\title{
La revue Matériaux et Techniques adopte le système de pagination à l'article
}

Avec l'avènement des nouvelles technologies et des médias électroniques, la diffusion de l'information véhiculée par ces médias ainsi que le référencement dans les bases de données deviennent une nécessité.

C'est pour cette raison et aussi avec le souci de mieux servir la communauté des Matériaux, que la revue Matériaux et Techniques change le système de pagination à partir de 2013. Ainsi, l'on passe d'une pagination « classique » (en numéros de pages) à un système plus flexible de numérotation à l'article.

À chaque article correspond désormais un numéro à 3 chiffres. Plus concrètement, en 2013, dans le volume 101, le dixième article du premier numéro de l'année sera référencé comme « Matériaux \& Techniques 101, 110 (2013) », le dixième article du deuxième numéro de l'année sera référencé comme « Matériaux E̛ Techniques 101, 210 (2013) », etc.

Les articles de la partie « Nouvelles scientifiques, techniques et industrielles » seront numérotés avec la lettre « N » (pour« Nouvelles ») suivi d'un numéro à deux chiffres. Ainsi, pour le volume 101, le troisième article des « Nouvelles » aura la référence : « Matériaux 8 Techniques 101, N03 (2013)».

Ce nouveau système de numérotation permet de publier en ligne un article plus rapidement (sans devoir attendre que le numéro soit complet). Le volume contenant les articles publiés de cette façon est rempli au fur et à mesure jusqu'à sa clôture, et la publication définitive de chaque article devient indépendante de celle des autres. En conséquence, les articles (et les auteurs) seront référencés plus vite et mieux dans les bases de données.

Nous sommes sûrs que ce nouveau système de numérotation améliorera la diffusion de l'information scientifique, et aidera à mieux référencer le travail de nos auteurs, par ailleurs toujours soigneusement expertisé et validé.

Ariana Fuga

Rédacteur en chef adjoint Matériaux \& Techniques 